\title{
Colour Stability of Veneering Composites after Accelerated Aging
}

Triantafillos Papadopoulos ${ }^{a}$

Aspasia Sarafianou ${ }^{\mathrm{b}}$

Andreas Hatzikyriakos ${ }^{c}$

\section{ABSTRACT}

Objectives: To investigate the colour stability of four indirect composite restorative materials after accelerated aging.

Methods: Four indirect composites (Gradia, Signum+, HFO and Adoro) were used. For each material, six specimens were prepared and subjected to accelerated aging (Suntest CPS+, Atlas, Chicago, IL, USA) according to ISO 7491. A Dr. Lange Microcolor Data Station colorimeter (Braive Instruments, Liege, Belgium) was used to measure specimen colour before and after aging. Measurements were performed according to the CIE L*a*b* system, and the mean $L^{*}, a^{*}$ and $b^{*}$ values for each material were calculated. The equation $\Delta \mathrm{E}=\left[\left(\Delta \mathrm{L}^{*}\right) 2+\left(\Delta \mathrm{a}^{*}\right) 2+\left(\Delta \mathrm{b}^{*}\right)^{2}\right]^{1 / 2}$ was used to measure the total colour change $(\Delta E)$, where $\Delta \mathrm{L}^{*}, \Delta \mathrm{a}^{*}$ and $\Delta \mathrm{b}^{*}$ are the differences in the respective values before and after aging. One-way ANOVA were used to determine statistically significant differences in $\Delta \mathrm{L}^{*}, \Delta \mathrm{a}^{*}, \Delta \mathrm{b}^{*}$ and $\Delta \mathrm{E}$.

Results: No statistically significant differences were found in $\Delta \mathrm{L}^{*}, \Delta \mathrm{a}^{*}, \Delta \mathrm{b}^{*}$ and $\Delta \mathrm{E}$ among the materials tested $\left(P \Delta L^{*}=.063 ; P \Delta a^{*}=.521 ; P \Delta b^{*}=.984\right.$ and $\left.P \Delta E=.408\right)$. After aging, Gradia specimens showed an increase in lightness $\left(\Delta L^{*}=0.36\right)$ and a green-yellow shift $\left(\Delta a^{*}=-1.18, \Delta b^{*}=0.6\right)$, while Signum + specimens exhibited an increase in lightness $\left(\Delta \mathrm{L}^{*}=0.5\right)$ and a green-blue shift $\left(\Delta \mathrm{a}^{*}=-0.9, \Delta \mathrm{b}^{*}=-\right.$ $0.45)$. HFO specimens exhibited an increase in lightness $\left(\Delta L^{*}=0.75\right)$ and a green-yellow shift $\left(\Delta a^{*}=-\right.$ $\left.1.3, \Delta b^{*}=0.06\right)$, and Adoro specimens exhibited an increase in lightness $\left(\Delta L^{*}=2.07\right)$ and a green-yellow shift $\left(\Delta a^{*}=-1.3, \Delta b^{*}=0.68\right)$.

Conclusions: Colour changes were found to be within accepted values of perceptibility and clinical acceptance after accelerated aging, and no statistically significant differences were found in $\Delta \mathrm{L}^{*}, \Delta \mathrm{a}^{*}$, $\Delta \mathrm{b}^{*}$ and $\Delta \mathrm{E}$ among the materials tested. (Eur J Dent 2010;4:137-142)

Key words: Colour stability; Indirect composites; Accelerated aging.

a Associate Professor, Department of Biomaterials, School of Dentistry, National and Kapodistrian University of Athens, Athens, Greece.

b DDS, PhD, Clinical Instructor, Department of Prosthodontics, School of Dentistry, National and Kapodistrian University of Athens, Athens, Greece. Associate Professor, Department of Prosthodontics, School of Dentistry, Aristotelian University of Thessaloniki, Thessaloniki, Greece.
Corresponding author:

Triantafillos Papadopoulos

Department of Biomaterials, School of Dentistry,

National and Kapodistrian University of Athens,

Thivon 2 Athens 115 27, Greece.

Phone: +2107461100

Fax: +2107240633

E-mail: trpapadadent.uoa.gr 


\section{INTRODUCTION}

Indirect veneering composite resins are being used with increasing frequency as a viable alternative to porcelain due to their improved mechanical properties, satisfactory aesthetic performance and ease of handling. However, long-term colour stability of these resins remains a matter of concern. ${ }^{1}$ Restorations in the oral cavity are exposed to a variety of factors, such as temperature fluctuations, humidity, light, food and beverages. Composite materials change their colour as a result of external factors, such as dietary or smoking habits. ${ }^{2,3}$ Internal discoloration in the deeper layers of the materials involves a variety of factors related to their composition. More specifically, the type of photo-initiator, nature of resin matrix, filler content and particle size distribution are related to colour stability. ${ }^{4,5}$

Colour stability is also influenced by the intensity and duration of polymerisation $n^{6,7}$ and, consequently, by the degree of conversion. ${ }^{8}$ In addition, factors such as heat, water, and exposure to ambient and UV radiation and food colorants have been reported to affect colour stability. ${ }^{9-13}$

In an attempt to simulate long-term intraoral colour performance of composites, several in vitro methods have been developed. These methods include storage in water, dark and dry conditions ${ }^{6}$ and exposure to visible or UV radiation or several food staining solutions. ${ }^{14,15}$

The purpose of the present study was to investigate the colour stability of four indirect composite restorative materials after accelerated aging. The testing hypothesis was that the colour parameters of the tested materials remain within a clinically acceptable level after accelerated aging.

\section{MATERIALS AND METHODS}

Four indirect light-cured restorative composites of $A 2$ dentin shade were tested (Table 1). A total of 24 specimens were fabricated, 6 specimens for each material, by filling a metal ring of $1.23 \mathrm{~mm}$ in height and $8.67 \mathrm{~mm}$ in diameter with the unset composite material, pressing it between two microscopic glass slides and covering it with transparent polystyrene matrix strips. The specimen dimensions allowed the use of a special specimen holder in the weathering device. For groups A, B, $C$ and $D$, the indirect composites Gradia, Signum+, HFO and Adoro, respectively, were used. The ma- terials were photo- and/or thermo-polymerised, according to the manufacturer's instructions. After removing the inhibition layer from one surface of each of the disc specimens, further polishing was performed with $\mathrm{SiC}$ paper up to 800 grit.

The specimens were aged immediately afterwards in a weathering device (Suntest CPS+, Atlas, Chicago, IL, USA) according to ISO 7491.16 Each aging cycle consisted of 20 min la dry period of $17 \mathrm{~min}$ followed by a 3-min rinse with deionised water at $37^{\circ} \mathrm{C}$ ). The total exposure time was $72 \mathrm{~h}$ under the following conditions: UV irradiation at 800-300 $\mathrm{nm}$ wavelength, $765 \mathrm{~W} / \mathrm{m}^{2}$ irradiance and $66 \mathrm{MJ} / \mathrm{m}^{2}$ daily radiant exposure.

A Dr. Lange Microcolor Data Station colorimeter (Braive Instruments, Liege, Belgium) was used to measure specimen colour before and after accelerated aging.

A custom-made specimen holder, fabricated from additional type silicone (Panasil, Kettenbach, Eschenburg, Germanyl, was used to standardise specimen orientation against the colorimeter head to achieve consistency in repeated measurements. This procedure also ensured a standard background and elimination of the influence of external light at specimen edges. After colorimeter calibration, measurements were performed according to the CIE L*a*b* system, and the mean $L^{*}, a^{*}$ and $b^{*}$ values for each material were calculated. The equation $\Delta E=\left[\left(\Delta L^{*}\right)^{2}+\left(\Delta a^{*}\right)^{2}+\left(\Delta b^{*}\right)\right.$ $\left.{ }^{2}\right]^{1 / 2}$ was used to determine the total colour change $(\Delta E)$, where $\Delta L^{*}, \Delta a^{*}$ and $\Delta b^{*}$ are differences in the respective values before and after aging. According to the most commonly accepted values of visual perceptibility ${ }^{17}$ and clinical acceptance, ${ }^{14}$ colour changes for composites were considered to be visually perceptible when $\Delta \mathrm{E}>1$ and clinically acceptable when $\Delta \mathrm{E}<3.3$.

One-way ANOVA were used to determine statistically significant differences in $\Delta \mathrm{L}^{*}, \Delta \mathrm{a}^{*}, \Delta \mathrm{b}^{*}$ and $\Delta \mathrm{E}$ among the materials tested. The type of the material was the independent variable. A level of statistically significant difference of $\alpha=.05$ was employed in all cases.

Statistical analyses were performed using SigmaStat software (Jandel, St. Rafael, CA, US).

\section{RESULTS}

The results of the colour measurements are presented in Table 2. No statistically significant differences were found in $\Delta \mathrm{L}^{*}, \Delta \mathrm{a}^{*}, \Delta \mathrm{b}^{*}$ and $\Delta \mathrm{E}$ among the materials tested. After accelerated aging, specimens of group A (Gradia) showed a low increase in lightness $\left(\Delta \mathrm{L}^{*}=0.36\right)$ and a predominantly green shift accompanied by a low yel- 
low shift $\left(\Delta a^{*}=-1.18, \Delta b^{*}=0.6\right)$, while specimens of group $B$ (Signum+) exhibited a low increase in lightness $\left(\Delta L^{*}=0.5\right)$ and a predominantly green shift accompanied by a low blue shift $\left(\Delta \mathrm{a}^{*}=-0.9\right.$, $\Delta \mathrm{b}^{*}=-0.45$ ). Specimens of group $\mathrm{C}(\mathrm{HFO})$ exhibited a low increase in lightness $\left(\Delta \mathrm{L}^{*}=0.75\right)$ and a predominantly green shift ( $\left.\Delta \mathrm{a}^{*}=-1.3, \Delta \mathrm{b}^{*}=0.06\right)$, while specimens of group $D$ (Adoro) predominantly exhibited an increase in lightness $\left(\Delta L^{*}=2.07\right)$ and a green shift $\left(\Delta \mathrm{a}^{*}=-1.3\right)$ accompanied by a low yellow shift $\left(\Delta b^{*}=0.68\right)$.

\section{DISCUSSION}

The CIE L*a*b* system is a widely used system that defines colour using three parameters, $L^{*}, a^{*}$ and $b^{*} \cdot{ }^{18} L^{*}$ corresponds to the value of an object, ranging from white $(+)$ to black (-). Both $a^{*}$ and $b^{*}$ are on the chromatic scale; a* measures red $(+)$ at one end and green (-) on the other, while $b^{*}$ measures yellow $(+)$ and blue (-), respectively. $\Delta \mathrm{E}$ represents the overall colour change that could be reported by an observer.

It is widely acknowledged $9,15,19,20$ that clinically acceptable $\Delta \mathrm{E}$ values are less than 3.3. According to the results of the present study, the testing hypothesis was verified: all tested materials exhibited $\Delta \mathrm{E}$ within clinically acceptable limits, with a deviation in measurements of Adoro (2.68 \pm 0.98 ). This result is in agreement with findings of other studies investigating similar materials. In a study by Stober et $\mathrm{al}^{12}$ in which the colour stability of veneering composites was assessed, $\Delta \mathrm{E}$ ranged between 1 and 3.3 for the majority of materials tested (Colombus, Artglass, Targis, Vita Zeta LC, BelleGlass) after $96 \mathrm{~h}$ of accelerated aging. Only Sinfony and Zeta $\mathrm{HC}$ exhibited $\Delta \mathrm{E}$ of less than 1. After $168 \mathrm{~h}$, all materials showed $\Delta \mathrm{E}$ of more than 3.3, with the exception of Vita Zeta HC, which showed visible but acceptable discoloration $(1<\Delta \mathrm{E}$ $<3.3$ ). The main increase was in the colorimetric value $b^{*}$, meaning that the specimens became yellowish. In a study involving 5 veneering composites (Conquest Sculpture, Thermoresin LC, Vita Zeta, Compo Plus, BelleGlass HP), Kolbeck et $\mathrm{al}^{10}$ found $\Delta E$ of 0.8 for Vita Zeta LC and 2.9 for BelleGlass HP after 72 h of UV irradiation. Most materials showed a yellow-green shift and became darker. The values of the yellow shifts were higher than those of the green shifts. Tanoue et $\mathrm{al}^{21}$ investigated the colour stability of Cesead and Newmetacolor Infinis and reported minimal colour changes ( $\Delta \mathrm{E}$ of 1.2 and 0.5 , respectively) after immersion in water for 4 weeks.

Table 1. Materials tested.

\begin{tabular}{lcc}
\hline Composites & Monomers & Fillers \\
\hline $\begin{array}{l}\text { Gradia } \\
\text { (GC Europe, N. V. Leuven, Belgium) }\end{array}$ & UDMA & $\begin{array}{c}\text { Microfine ceramic/ prepolymer filler, } \\
75 \% \mathrm{wt}\end{array}$ \\
\hline $\begin{array}{l}\text { Signum+ } \\
\text { (Heraeus Kulzer, GmbH, Hanau, Germany) }\end{array}$ & $\begin{array}{c}\text { Bis-GMA, } \\
\text { Bis-PMA, } \\
\text { UDMA }\end{array}$ & $\mathrm{ZrO}_{2}, \mathrm{SiO}_{2}, 78.5 \%$ wt \\
\hline HFO & Bis-GMA, \\
(GDF mbH, Roshbach, Germany) & UDMA & $\mathrm{BaO}_{2}, \mathrm{SiO}_{2}, 75 \%$ wt \\
\hline Adoro & TEGDMA & \\
(lvoclar-Vivadent,Schaan-Liechtenstein) & UDMA & Inorganic fillers, 65\% wt \\
\hline
\end{tabular}

Table 2. Mean values and standard deviation of $\Delta \mathrm{L}^{*}, \Delta \mathrm{a}^{*}, \Delta \mathrm{b}^{*}$ and $\Delta \mathrm{E}$.

\begin{tabular}{lcccc}
\hline Groups & $\Delta \mathrm{L}^{*}$ & $\Delta \mathrm{a}^{*}$ & $\Delta \mathrm{b}^{*}$ & $\Delta \mathrm{E}$ \\
\hline A (Gradia) & $0.36 \pm 0.19$ & $-1.18 \pm 1.1$ & $0.6 \pm 0.66$ & $1.73 \pm 0.71$ \\
B (Signum+) & $0.50 \pm 0.3$ & $-0.91 \pm 0.75$ & $-0.45 \pm 0.56$ & $1.35 \pm 0.57$ \\
C (HFO) & $0.75 \pm 0.83$ & $-1.33 \pm 0.61$ & $0.06 \pm 0.81$ & $1.75 \pm 0.9$ \\
D (Adoro) & $2.07 \pm 1.06$ & $-1.31 \pm 0.78$ & $0.68 \pm 0.33$ & $2.68 \pm 0.98$ \\
\hline
\end{tabular}

No statistically significant differences $\left(\mathrm{P} \Delta \mathrm{L}^{*}=.063 ; \mathrm{P} \Delta \mathrm{a}^{*}=.521 ; \mathrm{P} \Delta \mathrm{b}^{*}=.984\right.$ and $\left.\mathrm{P} \Delta \mathrm{E}=.408\right)$ were found among the materials tested. 
In a clinical study regarding the colour stability of veneering resins, Rosentritt et al ${ }^{22}$ reported that the materials tested (Visio-Gem, Dentacolor and Vita Zetal became darker and more yellow after 18 months of clinical performance $(\Delta E$ of 1-2.5). They attributed this change to UV stabilisers, whose action was obstructed by the accelerated aging procedure. Setz and Enge ${ }^{23}$ also reported a statistically significant yellow shift of facings made with Lucipast and Dentacolor after 2 years in the mouth. Matsumura et a ${ }^{24}$ tested the clinical performance of 110 restorations veneered with Cesead material and reported a «colour matching ability» (colour stability) of $82 \%$ after an observation period of 4 years and 5 months, $75.5 \%$ after 5 years and 3 months and $67.3 \%$ after 6 years and 3 months.

In this study, three materials (Adoro, HFO, Gradial showed a yellow shift (positive $\Delta b^{*}$ ). This phenomenon has been observed in previous studies and was attributed to the presence of residual camphorquinone, which is added to the composite materials as a photo-initiator. ${ }^{25,26}$ However, under the experimental conditions of the present study, intensive light during accelerated aging might have caused a further reaction of residual camphorquinone. Ferracane et $\mathrm{al}^{27}$ suggested that oxidation of unreacted double carbon bonds found in the polymerised resin, accelerated by UV light or heat in the presence of oxygen, leads to the formation of yellow-coloured peroxides. In addition, the by-products of tertiary aromatic amines laccelerators) may cause yellow-to-red-brown discoloration under the influence of light or heat., 7,28 According to Schulze et al, ${ }^{29}$ differences in the concentration and structure of photo-initiators and amines may explain the wide spectrum of $\Delta a^{*}$ and $\Delta \mathrm{b}^{*}$ values observed.

Matrix composition and filler type and size are thought to affect colour stability. ${ }^{19}$ During accelerated aging, specimens were rinsed with $37^{\circ} \mathrm{C}$ water for $3 \mathrm{~min}$ in every cycle, according to a specific program of the weathering device. Water can penetrate the matrix or matrix-filler interface..$^{30,31}$ As matrix content has been associated with water discoloration of composites, one would expect that a higher matrix-filler ratio would lead to increased discoloration. The nature of the matrix might influence water uptake, as TEGDMA absorbs more water than UDMA, ${ }^{32}$ which in turn absorbs less than Bis-GMA. ${ }^{33}$ In the present study, the findings for Adoro (low filler content $65 \%$ wt, $\Delta \mathrm{E}$ of 2.68 $\pm 0.98)$ are in accordance with these reports. It has been reported ${ }^{5,9}$ that small ratio differences could not account for variations in water sorption, which is the case for the other three materials tested. From the composition presentation of the tested materials in Table 1 and the results of $\Delta \mathrm{E}$ in Table 2 , it may be concluded that the material presenting the lowest $\Delta \mathrm{E}$ (Signum $+\Delta \mathrm{E}=1.35 \pm 0.57$ ) had the highest filler content $(78.5 \% \mathrm{wt})$, and its matrix is composed of Bis-GMA, Bis-PMA and UDMA.

When comparing Adoro with Gradia (similar matrix composition, different filler ratio), the above observations are verified: $\Delta \mathrm{E}$ of Gradia is lower. When Signum+ was compared to materials with similar filler content, the lowest $\Delta \mathrm{E}$ of Signum+ can be attributed to resin matrix composition differences. More specifically, Signum+ colour stability could be attributed to the presence of lower UDMA percentage combined with low-absorbing Bis-GMA and non-absorbing Bis-DMA ${ }^{34}$ compared to Gradia (UDMA only). The lower colour stability of HFO may be due to the presence of TEGDMA (higher water sorption).

In addition to matrix content and composition, filler size and distribution may also influence water discoloration, 9,19 presumably through hydrolytic degradation of the filler-matrix interface and modification of the way light is scattered by the particles. According to Dietschi et al, ${ }^{5}$ low water sorption, high filler-resin ratio, reduced particle size, hardness and an optimal filler-matrix coupling system are related to improved resistance to discoloration.

According to Schulze, ${ }^{29}$ accelerated aging is an extrinsic post-curing factor. High temperature during accelerated aging could have increased the degree of conversion, leading to a change in the refractive index of the matrix. This in turn would make the material less translucent, a result of increased scattering. ${ }^{35,36}$ According to Kolbeck et $\mathrm{al}^{10}$ the matrix composition combined with the quality of the polymerisation reaction is mainly responsible for the colour stability of veneering composites. According to Matsumura, ${ }^{24}$ colour stability is affected by the percentage of remaining $\mathrm{C}=\mathrm{C}$ bonds (\%RDB). It has also been shown that composites containing more than $35 \%$ of unconverted $\mathrm{C}=\mathrm{C}$ bonds tend to be highly susceptible to 
discoloration. ${ }^{37}$ In a recent study, however, ${ }^{38}$ no correlation was found between unconverted $\mathrm{C}=\mathrm{C}$ bonds and discoloration.

Colour difference $(\Delta E)$ evaluation using a colorimeter is a repeatable, sensitive and objective method. There are, however, some limitations concerning the use of colorimeters with different optical configurations as well as the "edge loss" phenomenon. Therefore, colour measurement may not be completely accurate. . $^{39,40}$

In the present study, a custom-made specimen holder was used to eliminate the "edge loss" phenomenon. In future studies, it would be useful to consider factors such as light scattering, gloss, shade and transparency when assessing overall discoloration of these materials. ${ }^{11,19}$

\section{CONCLUSIONS}

According to the results of the present study:

- No statistically significant differences were found in $\Delta \mathrm{L}^{*}, \Delta \mathrm{a}^{*}, \Delta \mathrm{b}^{*}$ and $\Delta \mathrm{E}$ values among the materials tested.

- The predominant colour change after accelerated aging was a green-yellow shift for Gradia $\left(\Delta a^{*}=-1.18, \Delta b^{*}=-0.6\right)$, a green-blue shift $\left(\Delta a^{*}=-\right.$ $\left.0.9, \Delta b^{*}=-0.45\right)$ for Signum+, an increase in lightness $\left(\Delta L^{*}=0.75\right)$ and green shift $\left(\Delta a^{*}=-1.3\right)$ for HFO and an increase in lightness $\left(\Delta \mathrm{L}^{*}=2.06\right)$ and greenyellow shift $\left(\Delta \mathrm{a}^{*}=-1.3, \Delta \mathrm{b}^{*}=0.6\right)$ for Adoro.

- Colour changes after accelerated aging were within clinically acceptable ranges $(\Delta \mathrm{E}<3.3)$ for all the materials tested.

\section{REFERENCES}

1. Lee YK, Lim B-S, Kim C-W, Powers JM. Color characteristics of low-chroma and high-translucence dental resin composites by different measuring modes. J Biomed Mater Res 2001;58:613-621.

2. Doray PG, Wang X, Powers JM, Burgess JO. Accelerated aging affects color stability of provisional restorative materials. J Prosthodont 1997;6:183-188.

3. Noie F, O' Keefe KL, Powers JM. Color stability of resin cements after accelerated aging. Int J Prosthodont 1995;8:5155.

4. Park YG, Chae KH, Rawls HR. Development of a new photoinitiator system for dental light-cured composite resins. Dent Mater 2000;15: 120-127.

5. Dietschi D, Campanile G, Holz J, Meyer JM. Comparison of the color stability of ten new-generation composites: an in vitro study. Dent Mater 1994:10:353-362.
6. Hosoya Y. Five-year color changes of light-cured resin composites: influence of light-curing times. Dent Mater 1999;15:268-274.

7. Janda R, Roulet JF, Latta M, Steffin G, Rüttermann S. Color stability of resin-based filling materials after aging when cured with plasma or halogen light. Eur J Oral Sci 2005;113:251-257.

8. Imazato S, Tarumi H, Kobayashi K, Hiraguri H, Oda K, Tsuchitani Y. Relationship between the degree of conversion and internal discoloration of light-activated composite. Dent Mater J 1995;14:23-31.

9. Vichi A, Ferrari M, Davidson CL. Color and opacity variations in three different resin-based composite products after water aging. Dent Mater 2004;20:530-534.

10. Kolbeck C, Rosentritt M, Reinhold L, Handel G. Discoloration of facing and restorative composites by UV-irradiation and staining food. Dent Mater 2006;22:63-68.

11. Lee YK, El Zawahry M, Noaman KM, Powers JM. Effect of mouthwash and accelerating aging on the color stability of esthetic restorative materials. Am J Dent 2000;13:159-161.

12. Stober T, Gilde H, Lenz P. Color stability of highly filled composite resin materials for facings. Dent Mater 2001;17:8794.

13. Bagheri R, Burrow MF, Tyas M. Influence of food simulating solutions and surface finish on susceptibility to staining of aesthetic restorative materials. J Dent 2005;33:389-398.

14. Ruyter IE, Nielner K, Möller BR. Color stability of dental composite resin materials for crown and bridge veneers. Dent Mater 1987;3:246-251.

15. Um CM, Ruyter IE. Staining of resin-based veneering materials with coffee and tea. Quintessence Int 1991;22:377386.

16. ISO 7491. Dental materials-Determination of colour stability. International Organization for Standardization. 2nd edition Geneva, Switzerland 2000.

17. Seghi RR, Hewlett ER, Kim J. Visual and instrumental colorimetric assessments of small color differences on translucent dental porcelain. J Dent Res 1989;68:1760-1764.

18. C.I.E. Commission Internationale de l'Eclairage. Recommendations on Uniform Color Spaces, Color Difference Equations and Metric Color Terms, in Suppl.No 2 to Pub. No 15, Paris: Bureau Central de la CIE; 1978.

19. Inokoshi S, Burrow MF, Kataumi M, Yamada T, Takatsu T. Opacity and color changes of tooth colored restorative materials. Oper Dent 1996b;21:73-80.

20. Kim HS, Um CM. Color differences between resin composites and shade guides. Quintessence Int 1996;27:559-567.

21. Tanoue N, Soeno K, Kawasaki K, Atsuta M. Influence of acidulated phosphate fluoride solution on the color stability of indirect composites. J Prosthet Dent 2004;92:343-347. 
22. Rosentritt M, Esch J, Behr M, Leibrock A, Handel G. In vivo color stability of resin composite veneers and acrylic resin teeth in removable partial dentures. Quintessence Int 1998;29:517-522.

23. Setz J, Engel E. In vivo color stability of resin-veneered telescopic dentures. A double blind pilot study. J Prosthet Dent 1997;77:486-491

24. Matsumura $H$, Nakamura M, Tanoue N, Atsuta M. Clinical evaluation of an urethane tetramethacrylate-based composite material as a prosthetic veneering agent. J Oral Rehabil 2000;27:846-852.

25. Shintani H, Yamaki M, Inoue T. Analysis of camphorochinone in visible light-cured composite resins. Dent Mater $1985 ; 1 ; 124-126$.

26. Eldiwany M, Friedl KH, Powers JM. Colour stability of lightcured and post-cured composites Am J Dent 1995;8:179181.

27. Ferracane JL, Moser JB, Greener EH. Ultraviolet light-induced yellowing of dental restorative resins. J Prosthet Dent 1985;54:483-487.

28. Janda R, Roulet JF, Kaminsky M, Steffin G, Latta M. Color stability of resin matrix restorative materials as a function of the method of light activation. Eur J Oral Sci 2004;112:280-285.

29. Schulze KA, Marshall SJ, Gansky SA, Marshall GW. Color stability and hardness in dental composites after accelerated aging. Dent Mater 2003;19:612-619.

30. Braden M, Clarke RL. Water absorption characteristics of dental microfine composite filling materials I. Proprietary materials. Biomater 1984;5:369-372.

31. Oysaed H, Ruyter IE. Water sorption and filler characteristic of composites for use in posterior teeth. $J$ Dent Res 1986;65:1315-1318.

32. Braden M, Clarke RL. Water absorption characteristics of dental microfine composite filling materials. II. Experimental materials. Biomater 1984;5:373-375.

33. Pearson GJ, Longman CM. Water sorption and solubility of resin- based materials following inadequate polymerization by a visible-light curing system. J Oral Rehabil 1989;16:57-61.

34. Kalachandra S, Terner DT. Water sorption of polymethacrylate networks: BIS-GMA/TEGDMA copolymers. J Biomed Mater Res 1987;21:329-338.

35. Lee YK, Powers JM. Color changes of resin composites in the reflectance and transmittance modes. Dent Mater 2007;23:259-264.

36. Yap AU, Sim CP, Loganathan V. Polymerization color changes of esthetic restoratives. Oper Dent 1999;24:306311.
37. Reinhardt KJ. Uncoverted double bonds and interface phenomena in composite materials. D Jahnartz Zeit 1991;46:204-208.

38. Sarafianou A, losifidou S, Papadopoulos T, Eliades G. Color stability and degree of cure of direct composite restoratives after accelerated aging. Oper Dent 2007;32:406-411.

39. Sham ASK, Chu FCS, Chai J, Chow TW. Color stability of provisional prosthodontic materials. J Prosthet Dent 2004;91:447-452.

40. Jonhston WM, Hesse NS, Davies BK, Seghi RR. Analysis of edge losses in reflectance measurements of pigmented maxillofacial elastomer. J Dent Res 1996;75:752-760. 